\title{
Note sur l'éboulement du Glacier du Tour (Haute-Savoie)
}

(14 Août 1949)

\section{The breaking up of the Tour Glacier}

\author{
PAR M. MESSINES DU SOURBIER

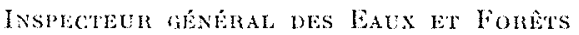



English synopsis p. 192

L'attention du public et du monde savant a été attirée cetle année par l'efrondrement subit du front d'un des glaciers les plus importants de la vallée de Chamonix : le glacier du Tour.

Le phénomène mérite d'être examiné en raison de son ampleur inusitée, et aussi de sa soudaineté, qui fut cause de nombreuses victimes.

\section{Historique du glacier du Tour. - Les mou- vements du front.}

Les caractéristiques du glacier du Tour sont les suivantes (approximativement) :

Bassin hydrologique. 1.530 ha dont 525 ha auA. dessus de $3.000 \mathrm{~m}$.

Surface du glacier... 829 ha (carte d'E.-M. 1895 ).

Débit de l'émissaire. 2 à $3 \mathrm{~m}^{3} / \mathrm{sec}$ en eau moyenne.

(selon Movgrn) 5 ì $9 \mathrm{~m}^{3} / \mathrm{sec}$ par temps de crue.

Ce glacier est étudié par le Service forestier depuis 1907. Déjà à cette époque, le front de ce glacier, débordant d'un plateau sur une falaise à pente forte, s'écroulait constamment (chutes de séracs) et son aspect variait sans cesse.

Des crues notables du glacier du Tour ont été étudiées vers 1643 , puis 1818 .

En 1818, le front s'étalait sur les prés, bordant le hameau du Tour. Depuis cette époque, on note :

- une régression de 1818 à 1878 ;
- après un stationnement de quelques années, une progression de 1884 ì 1892;

- un recul jusqu'en 1897;

- une légère progression en 1898;

$\rightarrow$ et un recul presque continu depuis lors, malgré quelques oscillations annuelles dans un sens ou dans l'autre et notamment une avancée assez forte autour de l'année 1920.

En 1949, le front se trouvait à la cote 2.000 environ. Ainsi depuis 1818 , soit plus de 120 ans, le glacier a remonté de 550 mètres.

\section{Les conditions particulières du relief.}

A l'emplacement de son front actuel, le glacier se trouvait à un changement de pente. En amont, la masse glaciaire repose dans une combe surcreusée, à pente faible, et qui débouche au sommet d'une énorme falaise rocheuse dont la hauteur tolale va en croissant de la rive droite a la rive gauche :

\section{Rive droite :}

Elle s'étend de la cote 1.700 a la cote 1.950 ; différence de niveau : $250 \mathrm{~m}$.

Rive gauche :

Elle s'étend de la cote 1.650 à la cote 2.100 environ; différence de niveau : $450 \mathrm{~m}$.

Au centre, la falaise a une hauteur d'environ 400 mètres. Elle est constituée par des roches moutonnées, de nature cristalline; elle n'est pas verticale, mais sa pente est considérable, de l'ordre de $100 \%$ à $125 \%$. 


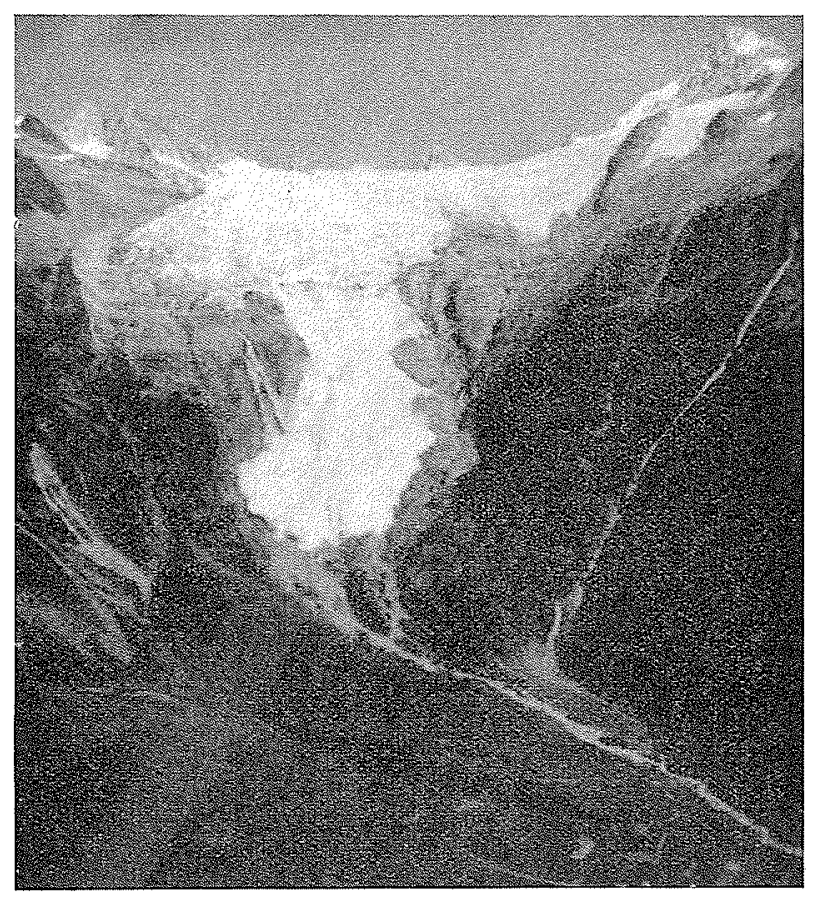

(Cliché Frison-Roche.)

Рното $\mathrm{x}^{\circ} 1$

PRISE PENDANT L'B́BOULEMENT DU CiLACAER DU TOUR (14 août 1949).

Cette muraiīle abrupte est burinée par les eaux et les glaces, el présente notamment en sa partie médiane et vers la rive gauche, un sillon plus fortement accusé dans lequel se logeait jusqu'à ces dernières années l'extrême-pointe de la langue terminale du glacier.

\section{L'explication du phénomène.}

Pendant la décade 1920-1930, et encore pendant les années suivantes, cette langue terminale du glacier se déplaçait, avançant ou reculant dans ce couloir et sur cette paroi abrupte. Elle franchissait done la zone du changement de pente (cote 2.000-2.100) et prenait appui dans le sillon, étayant en quelque sorte les masses de glace de l'amont.

Le glacier ne présentait aucune solution de continuité, sa pente longitudinale superficielle était dans l'ensemble assez régulière, et si, le long de l'immense front, des chutes de séracs étaient toujours prévisibles, celles-ci étaient du moins très localisées. Un effondrement de quelque importance n'était pas possible.

Un recul considérable a été subi par le glacier durant ces dernières années, et la combe où se logeait l'extrême pointe du glacier a été petit à petit abandonnée, sauf par quelques culots de slace morte.

Finalement, au cours de l'été 1949, la langue terminale, engagée dans le haut de l'abrupt, avait perdu son appui inférieur.

Les conditions topographiques étaient réunies pour que des chutes de séracs se produisent. Effectivement, il y en eut de fréquentes, comme cela s'était déjà produit autrefois, mais elles étaient peu importantes.

C'était devenu une attraction pour les touristes de passage au hameau du Tour.

Le 14 août, vers 16 heures, alors que, depuis le village, des personnes braquaient leurs jumelles sur le front du glacier en l'attente de ce spectacle original, la débâcle se produisit.

Elle a été fixće sur la pellicule par de nombreux alpinistes.

M. Frison-Roche : 2 clichés (photographie 1 et 2), pris en face du glacier du Tour, sous la montagne des Posettes, à $1.900 \mathrm{~m}$ d'altitude environ.

M. Benner, de la section du Haut-Rhin du C.A.F. : autres remarquables photos dans la Revue du Club.

M. le Docteur KLEIN, 65, boulevard Arago, à Paris: 5 clichés très intéressants, pris pendant le glissement.

Un alpiniste anglais : très belles photos au téléobjectif, avec détails extrêmement précis, qui paraîtront dans la Revue de Géographie alpine, avec un commentaire de J. Gurcronnet.

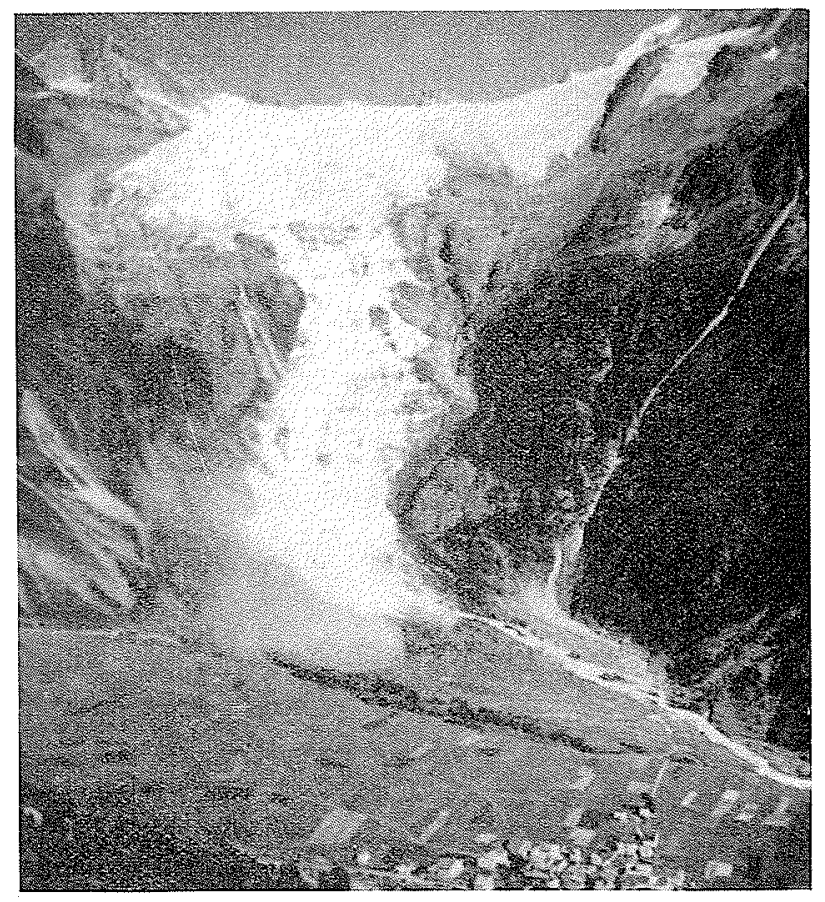

(Cliché Frison-Roche.)

PHOTO $\mathrm{N}^{\circ} 2$

PMISE PENDANT L'ÉBOULEMENT DU GLAGIER DU TOUR (14. août 1949). 


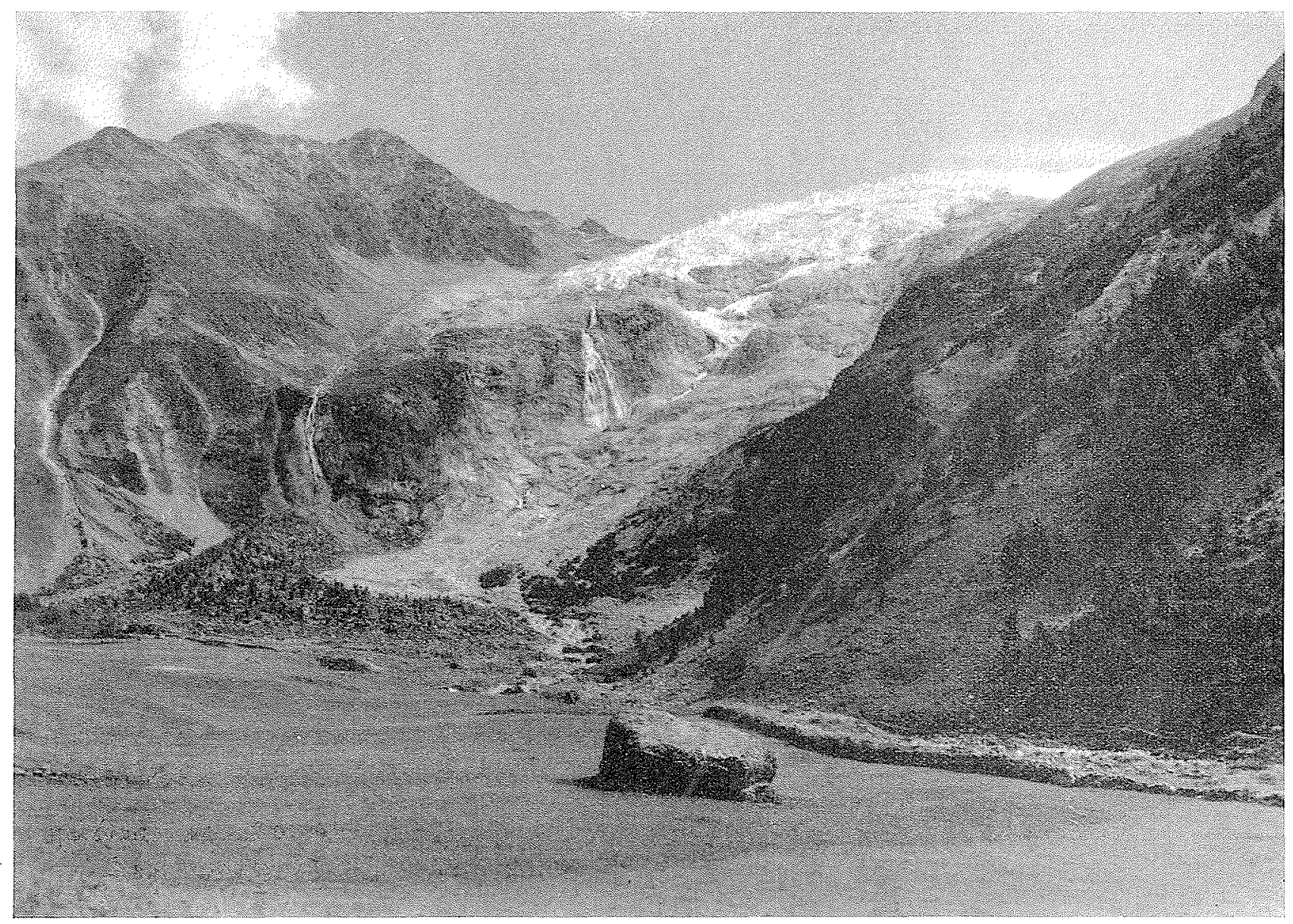

Photo $x^{6} 3$, - Le Glacher de Toun ex septembire 194.

(cliché Tuirraz.)

Ces opérateurs ont pu prendre des photographies tout à leur aise, au moment du décrochement, et avant même que la masse n'atteigne le fond de la vallée, car le phénomène a duré assez longtemps pour franchir une dénivellée de $400 \mathrm{ml}$ et s'étaler sur le cone de déjection du torrent. (Durée probable : 8 à 10 minutes.) (Voir observations Movgrn au sujet du même phénomène le 20 juillet 1910 .)

D'autre part, le photographe professionnel Tarraz, de Chamonix, a pris en septembre 19!9, pour le compte du Service des Eaux et Forêts, à partir du repère habituel, une photo du glacier a comparer avec les vues précédentes de 1915 , 1925, 1946 et 1948. Il a également pris, sur la demande de M. I'Inspecteur Bovverot, au téléobjectif, une photo du front du glacier où s'est produit le décrochement.

\section{Importance de l'éboulenient.} ron.

Largeur du décrochement : 200 mètres enviL'étalement de la gilace sur le cône de déjec- tions du torrent représente une surface de 12 hectares environ : $700 \mathrm{~m}$ de longueur sur une largeur variant de 100 a 250 mètres.

L'épaisseur de la glace broyée ou pulvérisce, mélangée de bloes morainiques, variait de $1 \mathrm{~m}$ à $20 \mathrm{~m}$.

Evaluation du volume de la glace ćboulée : 400 à $500.000 \mathrm{~m}^{3}$ (la presse régionale a annoncé 10 millions de $\left.\mathrm{m}^{3}\right)$.

\section{rictimes.}

Six promeneurs ont été ensevelis par l'avalanche. Les corps de trois d'entre eux ont éte retrouvés les 16 aoùt, 18 et 19 septembre.

$$
\therefore
$$

Cet effondrement, cette chute de glace n'a rien de commun avec la véritable débâcle glaciaire, résultant de la rupture de poches d'eau intraglaciaires.

De varaies débâcles de l'espèce ont été notées :

Téte Rousse : la catastrophe célébre de 1892; 
Glacier d'Argentièes : 17 juin 1904;

Les Bossons : 14 mai 1920 ;

La Mer de Glace : 25 septembre 1920.

Cette dernière débâcle était due à une crue de l'Arveyron et à des obstructions sous-glaciaires du torrent en crue,

Rien de pareil au glacier du Tour. La « débâcle » signalée par Movgis, le 20 juillet 1910 , au Tour et dont Mougrn lui-mème a été le témoin, n'a pas été autre chose qu'une chute de séraes, répétée à deux reprises à $15 \mathrm{~h} .15 \mathrm{et}$ ¿' 17 h. 24, comme l'a soigneusement noté l'ancien Inspecteur général des Eaux et Forêts. La durée du phénomène, notée également, a ćlé chaque fois de 7 minutes.

\section{Les conditions de l'enneigement au Tour.}

Nous avons la bonne fortune de posséder, pour la vallée de Chamonix et les aboids, de nombreuses stations d'observations nivométriques (lables à neige) fonctionnant depuis de nombreuses annees :
I.es Contamines, les Houches, Chamonix-Ville (Le Bouchet) el le Tour lui-même.

Le tableau ci-apres donne les moyennes nivométriques décennales de 1900 à 1949, aux stations du Bouchet et du Tour :

\begin{tabular}{|c|c|c|c|c|}
\hline \multirow{2}{*}{ Périodes } & \multicolumn{2}{|c|}{$\begin{array}{l}\text { Chamonix } \\
\text { (Le Bouchet) } \\
\text { (alt. } 1.050 \mathrm{~m} \text { ) }\end{array}$} & \multicolumn{2}{|c|}{$\begin{array}{c}\text { Le Tour } \\
\text { (altitude } 1.431 \mathrm{~m} \text { ) }\end{array}$} \\
\hline & $\begin{array}{c}\text { Hautenr } \\
(\mathrm{m})\end{array}$ & $\begin{array}{l}\text { Nombre } \\
\text { de jours }\end{array}$ & $\begin{array}{c}\text { Hauteur } \\
(\mathrm{m})\end{array}$ & $\begin{array}{l}\text { Nombre } \\
\text { de jours }\end{array}$ \\
\hline $1900-1910$ & $2,85(1)$ & $36(1)$ & 9,45 & 77 \\
\hline $1910-1920$ & 3,97 & 46 & $11,27(2)$ & $89(2)$ \\
\hline $1920-1930$ & 3,37 & 44 & 7,93 & 74 \\
\hline $1930-1940$ & 2,94 & 40 & 8,38 & 66 \\
\hline $1940-1949$ & 2,86 & 41 & 7,54 & 57 \\
\hline $\begin{array}{l}\text { Moyenne } \\
1900-1949 .\end{array}$ & $3,20(1)$ & $42(1)$ & $9, \quad(2)$ & $72(2)$ \\
\hline
\end{tabular}

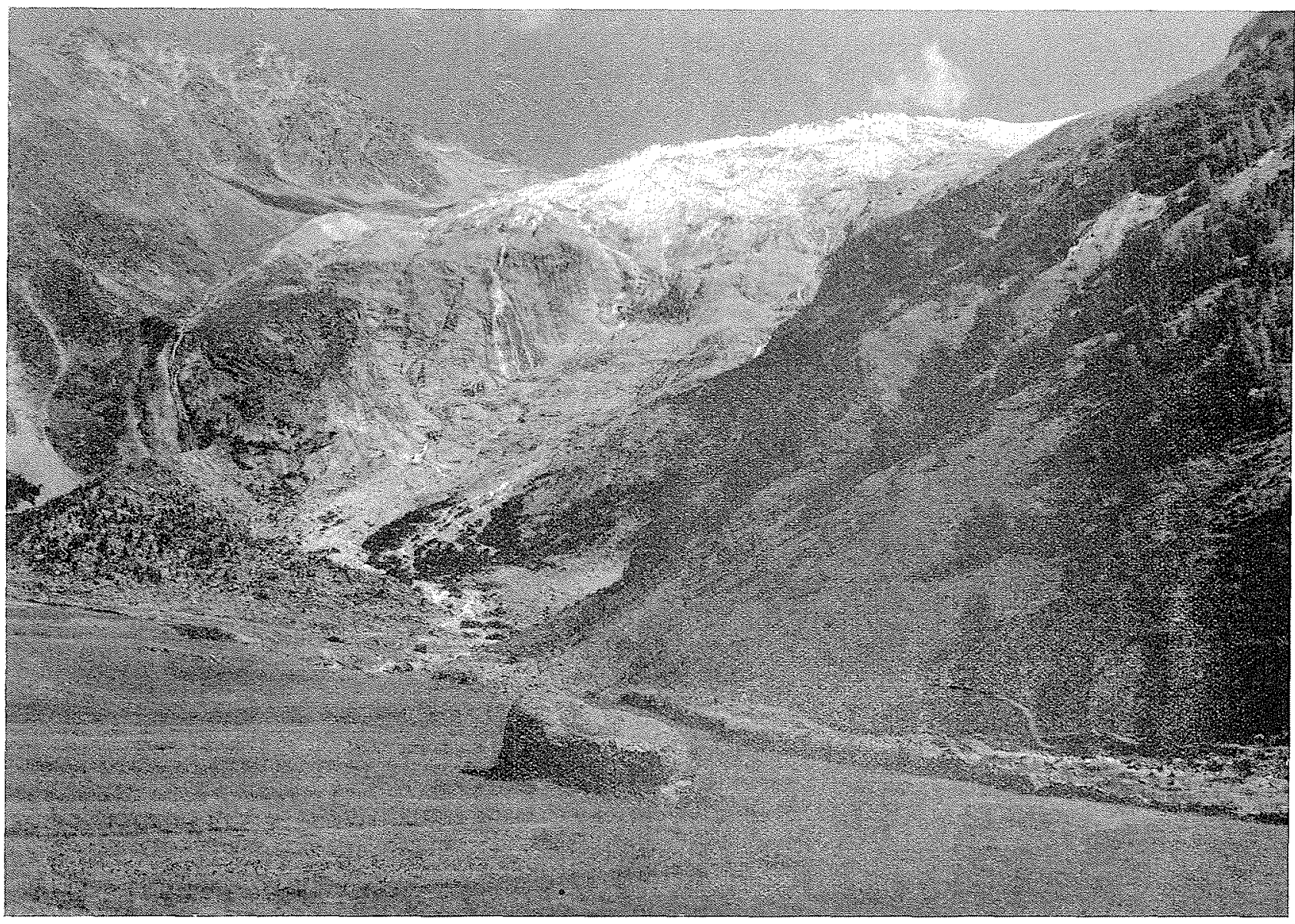




L'enneigement au Tour est exceptionnel : c'est le plus élevé enregistré dans une station habitée des Alpes françaises. On soupçonne, d'après ces chifferes, l'importance de l'enneigement à 2.000 et 3.000 mètres d'altilude.

La période 1910-1920 a été la plus enneigée; puis viennent les périodes 1900-1910, 1930-1940, 1920-1930; enfin la période la plus récente, 19401949 , est la moins enneigée.

Le maximum d'enneigement a eu lieu pendant l'hiver 1921-1922 (15,20 $\mathrm{m}$ et 98 jours); le minimum, l'hiver 1948-1949 (3,70 m et 34 jours).

L'avancée assez forte du glacier, constatéc en 1920-1921, coïncide avec l'enneigement maximum de la période de dix années qui venait de finir.

La pénurie des précipitations neigeuses de la décade en cours, surtout depuis l'hiver 1945-1946, et son accentuation encore plus marquée pendant les deux derniers hivers 1947-1948 et 1948-1949 ne sont évidemment pas étrangères à la fonte accélérée des glaciers et aux régressions considérables des langues terminales.

Or, on sait que la décrue d'un glacier, causée principalement par une pénurie de l'alimentation du névé, ne se répercute qu'au bout de quelques années sur le dissipateur. Ce serait donc l'enmeigement médiocre des années 1937 à 1940 notamment, puis celles de 1941 à 1943 qui se. raient actuellement en cause.

La médiocrité persistante de l'enneigement, se traduisant actuellement par des minima jamais égalés, durant les cinquante dernières années, permet de conclure que le recul des glaciers ira encore en s'amplifiant durant les prochaines années, même si les hivers à venir sont plus copieusement arrosés.

Pendant la campagne 1948-49, la pénurie des précipitations a été particulièrement marquée durant l'hiver.

En effet, les relevés des pluviomètres de Chamonix et du Tour domnent, pour chaque mois, et pour l'année météorologique entière, les résultats ci-après :

\begin{tabular}{|c|c|c|c|}
\hline Mois & $\begin{array}{l}\text { Chamonix } \\
\text { (Le Bouchet) } \\
\text { (alt. } 1.050 \mathrm{~m} \text { ) }\end{array}$ & $\begin{array}{c}\text { Le Tour } \\
\text { (alt. } 1.431 \mathrm{~m})\end{array}$ & Observations \\
\hline $\begin{array}{l}1948 \\
\text { Septembre: } \\
\text { Octobre : } \\
\text { Novembre: } \\
\text { Décembre : } \\
1949 \\
\text { Janvier : } \\
\text { Février : } \\
\text { Mars : } \\
\text { Avril : } \\
\text { Mai : } \\
\text { Juin : } \\
\text { Juillet : } \\
\text { Aon̂t : } \\
\text { Lame d'eau } \\
\text { annuelle: }\end{array}$ & $\begin{array}{c}135,75 \mathrm{~mm} \\
46,25 \\
100,75 \\
42,70 \\
\\
\\
60,80 \\
14,60 \\
30,20 \\
76,15 \\
142,75 \\
94,75 \\
37,00 \\
126,30 \\
\\
908,00 \mathrm{~mm}\end{array}$ & \begin{tabular}{|c|}
$127,3 \mathrm{~mm}$ \\
58,3 \\
100,7 \\
39,2 \\
\\
\\
72,2 \\
24,1 \\
52,4 \\
107,6 \\
138,2 \\
101,7 \\
71,6 \\
121,7 \\
\\
$1.015,0 \mathrm{~mm}$
\end{tabular} & 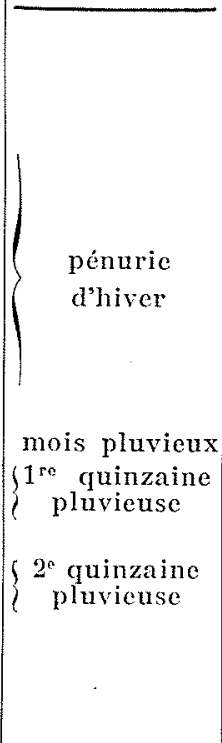 \\
\hline
\end{tabular}

dont environ le quart sous forme de neige.

On note d'une part l'indigence des neiges en hiver, suivie d'une pluviosité assez abondante au printemps, du $1^{\mathrm{er}}$ mai au 15 juin, puis de nouveau, la sécheresse qui dure jusqu'au 15 août, c'est-à-dire jusqu'à la catastrophe du Tour.

A cette époque, 14 août, la chaleur n'avait rien d'exagéré. En août, les maxima de température oscillent entre $14^{\circ}$ et $26^{\circ}$, et les minima entre $1^{\circ}$ et $13^{\circ}$.

Le 14 août, le maximum atteint $16^{\circ}$ et le minimum $1^{\circ}$.

Il n'y a dans ces chiffres de température rien d'excessif ni d'anormal, et ce n'est pas là qu'il faut chercher une des causes de l'éboulement.

O'n notera seulement que le temps, d'une manière générale, était très beau, le ciel sans nuages pendant de longues périodes de l'été, et l'insolation au maximum, ce qui a dû avoir pour effet d'accélérer la fusion des glaces, d'élargir et d'allonger les crevasses, qui effectivement étaient importantes à l'extrémité du front du glacier, en août 1949. Ceci, avec les conditions de relief, explique la chute fréquente de séracs. 